\title{
Protocol
}

\section{Whole-Cell Patch Recording from Drosophila Larval Neurons}

\author{
Richard Marley and Richard A. Baines
}

\section{INTRODUCTION}

The fruit fly Drosophila melanogaster has been instrumental in expanding our understanding of early aspects of neural development. The use of this model system has greatly added to our knowledge of neural cell-fate determination, axon guidance, and synapse formation. It has also become possible to access and make electrophysiological recordings directly from neurons in situ in an intact central nervous system (CNS), which has facilitated studies of the development and regulation of neuronal signaling. This protocol describes a procedure for revealing larval motor neurons and applying whole-cell patch recording techniques to these cells. The useful lifetime of first-instar larval preparations is $\sim 30 \mathrm{~min}$, and that of third-instar CNS preparations is up to $1 \mathrm{~h}$. It is therefore recommended that fresh preparations are used and that no breaks are taken during the procedure, although there may be time to pull and polish a patch pipette.

\section{RELATED INFORMATION}

Basic electrophysiological theory is not covered here and it is assumed that the reader has some prior experience. Protocols are available for Dissection of First- and Second-Instar Drosophila Larvae for Electrophysiological Recording from Neurons: The Flat (or Fillet) Preparation (Marley and Baines 2011a) and Dissection of Third-Instar Drosophila Larvae for Electrophysiological Recording from Neurons (Marley and Baines 2011b).

\section{MATERIALS}

RECIPES: Please see the end of this article for recipes indicated by $<\mathbf{R}>$.

It is essential that you consult the appropriate Material Safety Data Sheets and your institution's Environmental Health and Safety Office for proper handling of equipment and hazardous materials used in this protocol.

\section{Reagents}

Drosophila external saline $<\mathbf{R}>$

Drosophila larval CNS, isolated as in Dissection of First- and Second-Instar Drosophila Larvae for Electrophysiological Recording from Neurons: The Flat (or Fillet) Preparation (Marley and Baines 2011a) or Dissection of Third-Instar Drosophila Larvae for Electrophysiological Recording from Neurons (Marley and Baines 2011b)

Internal patch solution as appropriate for given experiment Patch pipette dye

Prepare 5- $\mu$ L aliquots of $0.4 \%$ (w/v) Alexa Fluor 488 hydrazide (sodium salt; Invitrogen A-10436) by dissolving 1 $\mathrm{mg}$ in $250 \mathrm{~mL}$ of an appropriate internal patch solution. Store frozen until needed. To make a working solution, add a further $155 \mu \mathrm{L}$ of the appropriate internal patch solution to an aliquot of Alexa Fluor 488.

Protease type XIV (Sigma P5147-5G)

Adapted from Drosophila Neurobiology (ed. Zhang et al.).

CSHL Press, Cold Spring Harbor, NY, USA, 2010.

Cite as: Cold Spring Harb Protoc; 2011; doi:10.1101/pdb.prot065664

www.cshprotocols.org 


\section{Equipment}

Anotop 10 syringe filters (0.2- $\mu$ m pore size; $10-\mathrm{mm}$ diameter) (Whatman 6809-1022)

Electrophysiology rig (including an upright compound microscope [e.g., Olympus BX51WI] with $10 \times$ air and $60 \times$ long-distance water-immersion lenses and $12.5 \times$ eyepieces)

The setup and maintenance of a rig is beyond the scope of this protocol; it is recommended that the reader look to more experienced colleagues for guidance.

Enzyme pipettes (borosilicate glass; 1 -mm outer diameter [OD], 0.78-mm inner diameter [ID] with filament) (Harvard Apparatus 30-0038)

MicroFil 28 AWG needles (World Precision Instruments MF28G67-5)

Microforge

Micropipette puller (e.g., P-97 Flaming/Brown; Sutter Instrument Co.)

Minisart 0.20- $\mu \mathrm{m}$ single-use hydrophilic syringe filters (Sartorius 16534)

Patch pipettes (borosilicate glass; 1-mm OD, 0.58-mm ID with filament) (Harvard Apparatus 30-0019)

Software suitable for data capture and analysis in patch-clamp experiments (e.g., pClamp from Molecular Devices)

Syringes (1-mL and 10-mL)

\section{METHOD}

\section{Preparation of Enzyme and Patch Pipettes}

1. Pull enzyme and patch pipettes.

Enzyme pipettes are patch-type electrodes with an opening of $\sim 5 \mu \mathrm{m}$. Patch pipettes have an opening, before polishing, of $\sim 1 \mu \mathrm{m}$.

2. Fire-polish the patch (but not enzyme) pipettes with the microforge to reduce tip diameter.

When filled with patch solution, the desired resistance is $\sim 15 \mathrm{M} \Omega$ for first-instar larvae and $\sim 10 \mathrm{M} \Omega$ for third-instar larvae.

\section{Technique to Reveal Larval Motor Neurons}

3. Under low magnification, ensure that the CNS is centered in view. Confirm that the CNS is also centered under high magnification. Perform a last check on CNS integrity. Check that no damage has been sustained during the dissection.

Almost any damage to a first-instar larval CNS will render it unusable.

4. Place an enzyme pipette containing a solution of $1 \%(\mathrm{w} / \mathrm{v})$ protease type XIV in Drosophila external saline in the electrode holder on the rig.

Enzyme pipettes are best filled using a 1-mL syringe with an Anotop 10 0.2- $\mu \mathrm{m}$ filter and a MicroFil 28 AWG needle. Only the tip of the electrode should be filled. The filter is useful to prevent debris from entering the pipette and blocking the tip.

5. Under low magnification, move the pipette gently, but swiftly, down to just above the CNS. Do not move the pipette below the field of view; always move the plane of focus and then move the pipette to match.

The CNS is very fragile and easily torn by the sharp edge of a pipette.

6. Repeat Step 5 under high magnification until the enzyme pipette is positioned 10-20 $\mu \mathrm{m}$ above the CNS.

7. Select an area along the dorsal midline (between the transverse nerves) and remove the glial sheath:

i. Gently, via a mouth pipette, suck a small section of the outer glial sheath into the tip of the enzyme pipette.

ii. Hold for $\sim 10 \mathrm{sec}$ to allow the enzyme to work, and then gently exhale.

iii. Continue this process until a rupture occurs in the sheath and the neurons are clearly seen. At first, your disruption of the glia may be overvigorous and will cause unintentional damage, but practice makes perfect. If suction is too hard, neurons will be removed from the preparation via the mouth pipette. 
Similarly, if the operator exhales with too much force, the CNS will be inflated like a balloon, likely disrupting many synaptic connections in the neuropil. First-instar preparations effectively have only a single layer of glial tissue to remove, whereas third-instar preparations have multiple layers that can require multiple applications of enzyme to remove. Neurons should be clearly visible with sharply defined edges. If they appear to be "fuzzy," then there is still glia wrapping to be removed.

\section{Patch Recording from Larval Motor Neurons}

8. Prepare the pipette for recording:

i. Fill the tip of a polished patch pipette with patch pipette dye either by back filling or by injecting using a 1-mL syringe with a MicroFil 28 AWG needle.

This enables cell identification after patch recording.

ii. Fill the rear half of the pipette with an appropriate Drosophila internal patch solution using the MicroFil 28 AWG needle attached to a 10-mL syringe and filtered by a Minisart 0.2- $\mu \mathrm{m}$ filter.

iii. Place the filled micropipette into the electrode holder, positioning its tip just above the preparation as for the enzyme pipette (see Steps 5 and 6).

The tip of the pipette is very narrow and therefore prone to blockage from free-floating debris remaining from the application of protease to the glial sheet. Take care and use extra speed to prevent this from occurring. If any blockage does occur, discard the pipette and repeat this step until an unblocked patch pipette is in position.

9. Before attempting to patch, check the electrode resistance and discard if it lies outside the 10-to 20-M $\Omega$ range (for first- or second-instar larvae) or the 5- to15-M $\Omega$ range (for third-instar larvae).

10. Select a motor neuron to patch.

The UAS/GAL4 expression system driving green fluorescent protein (GFP) in selected motor neurons will help when starting; for example, RN2-GAL4 drives expression in the aCC and RP2 motor neurons (Fujioka et al. 2003). A full description of how to record from neurons using a patch electrode is beyond the scope of this protocol. We strongly suggest that interested individuals seek help from established electrophysiology groups in their own institutions. Many electrophysiology amplifier manufacturers also provide tutorials for novice users. Patch recording from Drosophila neurons is no different from any other neuron, and so guidance can be obtained from any existing electrophysiology group.

11. After a recording, do not immediately change pipettes. Instead, switch to a GFP filter (440- to 480-nm excitation) and view the recorded Alexa Fluor dye-filled neuron under fluorescent light.

This will allow visualization of the recorded neuron; its dendritic morphology and muscle target (if a motor neuron) can be clearly observed.

12. Change patch pipettes and select a new neuron to record from.

First-instar larval preparations will remain viable only for up to $30 \mathrm{~min}$ from when the cuticle is first opened, limiting the number of recordings that can be taken. Two good recordings per first-instar preparation is an acceptable aim. However, a third-instar larval preparation remains viable for much longer and three or four good recordings per preparation may be made as long as suitable cells are present.

\section{REFERENCES}

Fujioka M, Lear BC, Landgraf M, Yusibova GL, Zhou I, Riley KM, Patel $\mathrm{NH}$, Jaynes JB. 2003. Even-skipped, acting as a repressor, regulates axonal projections in Drosophila. Development (Camb) 130: 5385-5400.

Marley R, Baines RA. 2011a. Dissection of first- and second-instar Drosophila larvae for electrophysiological recording from neurons:
The flat (or fillet) preparation. Cold Spring Harb Protoc doi: $10.1101 /$ pdb.prot065649.

Marley R, Baines RA. 2011b. Dissection of third-instar Drosophila larvae for electrophysiological recording from neurons. Cold Spring Harb Protoc doi: $10.1101 /$ pdb.prot065656. 


\section{RECIPE}

Recipes for items marked with $<\mathrm{R}>$ are provided here. Additional recipes can be found online at http:// www.cshprotocols.org/recipes.

\section{Drosophila external saline}

$135 \mathrm{~mm} \mathrm{NaCl}$

$5 \mathrm{~mm} \mathrm{KCl}$

$4 \mathrm{mM} \mathrm{MgCl} 2 \cdot 6 \mathrm{H}_{2} \mathrm{O}$

$2 \mathrm{mM} \mathrm{CaCl}_{2} \cdot 2 \mathrm{H}_{2} \mathrm{O}$

$5 \mathrm{~mm}$ TES ( $N$-tris[hydroxymethyl]methyl-2-aminoethane sulfonic acid)

$36 \mathrm{~mm}$ sucrose

If the calcium chloride and sucrose are omitted, then a $10 \times$ stock solution can be prepared that will last for months when kept refrigerated. We usually make a $500-\mathrm{mL}$ working solution $\left(50 \mathrm{~mL}\right.$ stock $\left.+450 \mathrm{~mL} \mathrm{H}_{2} \mathrm{O}\right)$, to which we add the required amount of calcium and sucrose. The $\mathrm{pH}$ (of the $10 \times$ stock solution) should be adjusted to 7.15 with $10 \mathrm{M}$ $\mathrm{NaOH}$. 


\section{Whole-Cell Patch Recording from Drosophila Larval Neurons}

Richard Marley and Richard A. Baines

Cold Spring Harb Protoc; doi: 10.1101/pdb.prot065664

\begin{tabular}{|c|c|}
\hline $\begin{array}{r}\text { Email Alerting } \\
\text { Service }\end{array}$ & Receive free email alerts when new articles cite this article - click here. \\
\hline $\begin{array}{r}\text { Subject } \\
\text { Categories }\end{array}$ & $\begin{array}{l}\text { Browse articles on similar topics from Cold Spring Harbor Protocols. } \\
\text { Developmental Biology ( } 728 \text { articles) } \\
\text { Drosophila ( } 272 \text { articles) } \\
\text { Electrophysiology ( } 104 \text { articles) } \\
\text { Imaging Development ( } 255 \text { articles) } \\
\text { Imaging for Neuroscience (342 articles) } \\
\text { Isolation (34 articles) } \\
\text { Patch Clamping (64 articles) }\end{array}$ \\
\hline
\end{tabular}

